\title{
Segmentation and Detection of Diabetic Retinopathy Exudates
}

\author{
A. Elbalaoui \\ Faculty of Science and \\ Technology \\ Beni-Mellal, Morocco.
}

\author{
M. Fakir \\ Faculty of Science and \\ Technology \\ Beni-Mellal, Morocco.
}

\author{
A. Merbouha \\ Faculty of Science and \\ Technology \\ Beni-Mellal, Morocco
}

\begin{abstract}
Diabetic retinopathy, the most common diabetic eye disease, occurs when blood vessels in the retina change. Sometimes these vessels swell and leak fluid or even close off completely. In other cases, abnormal new blood vessels grow on the surface of the retina. Early detection can potentially reduce the risk of blindness. This paper presents an automated method for the detection of exudates in retinal color fundus images with high accuracy, First, the image is converted to HSI model, after preprocessing possible regions containing exudate, the segmented image without Optic Disc (OD) using algorithm Graph cuts, Invariant moments $\mathrm{Hu}$ in extraction feature vector are then classified as exudates and non-exudates using a Neural Network Classifier. All tests are applied on database DIARETDB1.
\end{abstract}

\section{General Terms}

Color Image, Image processing

\section{Keywords}

Segmentation; Diabetic retinopathy; Graph cuts; Neural Network.

\section{INTRODUCTION}

In general, manual segmentation methods are very timeconsuming. Therefore, our focus is on semi-automatic and fully automatic methods. We concentrate on the minimization of user interaction in order to keep things as simple as possible while providing the results as fast as possible. To achieve this goal, we take advantage of latest developments in computer graphics hardware for noticeable performance speedups.

Medical Image Segmentation is the process of automatic or semi-automatic detection of boundaries within a $2 \mathrm{D}$ or $3 \mathrm{D}$ image. Image Segmentation is a process for dividing a given image into meaningful regions with homogeneous properties.

Many techniques have been employed for the exudate detection. The thresholding and region growing technique are widely used. Gardner et al. [2] proposed an automatic detection of diabetic retinopathy using an artificial neural network. The exudate identified from grey level images. Sinthaniyothin [3] uses maximum variance to obtain the optic disk center and a region growing segmentation method to obtain the exudates. Sanchez et al. [4] combine color and sharp edge features to detect exudates. Kavitha et al. [5] proposed median filtering and morphology operation for blood vessels detection. AkaraSopharak et al [6] reported the result of an automated detection of exudates from low contrast digital images of retinopathy patients with non-dilated pupils by Fuzzy C-Means clustering. Welfer, Scharcanski et al. [7], proposed a method based on mathematical morphology. They used the lightness $\mathrm{L}$ of the perceptually uniform Luv color space due to intensity fluctuations in the L channel are smaller than in the RGB. Akarasopharak et al. [8] proposed a series of experiments on feature selection and exudates classification using naive Bayes and Support Vector Machine (SVM) Classifiers. DeepashreeDevaraj et al. [9] proposed an automatic detection of diabetic retinopathy using gray scale morphology are identified. By considering macular region, Diabetic Retinopathy is classified into mild, moderate and severe conditions.

A major difficulty of medical image segmentation is the high variability in medical images. The techniques presented in this paper can be classified into three categories:

- Assessment and improvement of the image quality.

- Segmentation of exudates.

- Recognition.

The paper is organized as follows: Section II describe the Methodology used. Section III deals with the result and Discussion. The paper is ended by a conclusion.

\section{METHODOLOGY}

\subsection{Image Acquisition}

In this work, the input images used obtained from the DIAREDB1 database [1]. It consists of 89 color fundus images of $1500 \times 1152$ pixels of which 84 contain nonproliferative signs of the diabetic retinopathy, and 5 are considered as normal. Images were captured using the same 50 degree field-of-view digital fundus camera with varying imaging settings. This data set is referred to evaluate the performance of this method.

\subsection{Preprocessing}

After the acquisition of the image recognition system begins with the preprocessing method comprising the following functions: - RGB to HSI Conversion, Median filtering and Adaptive Histogram Equalization.

Therefore, our process that preprocessing fit is represented by the following diagram (figure 1). 


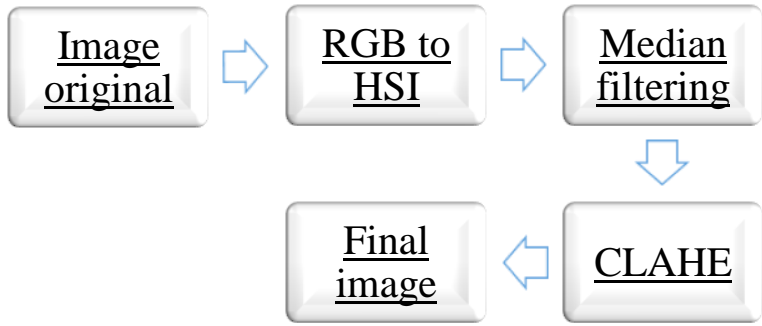

Fig 1: Preprocessing steps

\subsubsection{RGB to HSI Conversion}

First, we convert RGB color space image to HSI space by normalizing the range of the $\mathrm{R}, \mathrm{G}$, and $\mathrm{B}$ components to the interval from 0 to 1 .

Then we compute the values $r, g, b$ that are independent of intensity:

$r=\frac{R}{R+G+B}, g=\frac{G}{R+G+B}, b=\frac{B}{R+G+B}$

Each normalized H, S and I components are then obtained

$$
\begin{gathered}
\cos (H)=\frac{2 \mathrm{R}-\mathrm{G}-\mathrm{B}}{2 \sqrt{(R-G)^{2}-(\mathrm{R}-\mathrm{B})(\mathrm{G}-\mathrm{B})}}(2) \\
S=1-\frac{3}{R+G+B} * \min (R, G, B) \\
I=\frac{R+G+B}{3} \text { (4) }
\end{gathered}
$$

To separate the color components with intensity and brightness, the component retinal input images in the RGB color space are converted to the HSI color space (Figure 2).

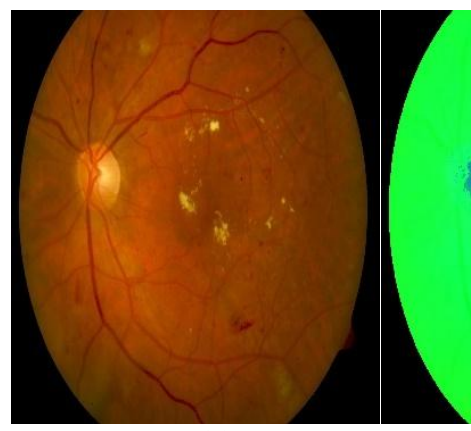

(a)

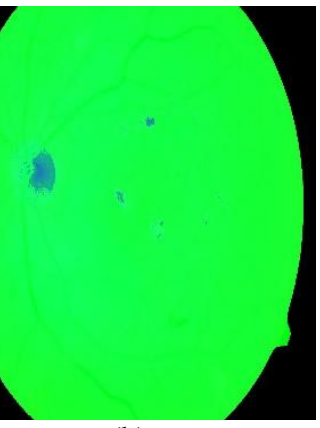

(b)
Fig 2: (a) testing image, (b) RGB to HSI space

\subsubsection{Median filtering}

In signal processing, it is often desirable to be able to perform some kind of noise reduction on an image or signal. The median filter is a nonlinear digital filtering technique, often used to remove noise. Such noise reduction is a typical preprocessing step to improve the results of later processing. Median filtering is very widely used in digital image processing because, under certain conditions, it preserves edges while removing noise. To evenly distribute the intensity of our database ofimages, the component I of the HSI color space is extracted (Figure 2 (b)) and filtered through a $3 \times 3$ median filter, illustrate the result after using median filter (Figure 2 (a)).

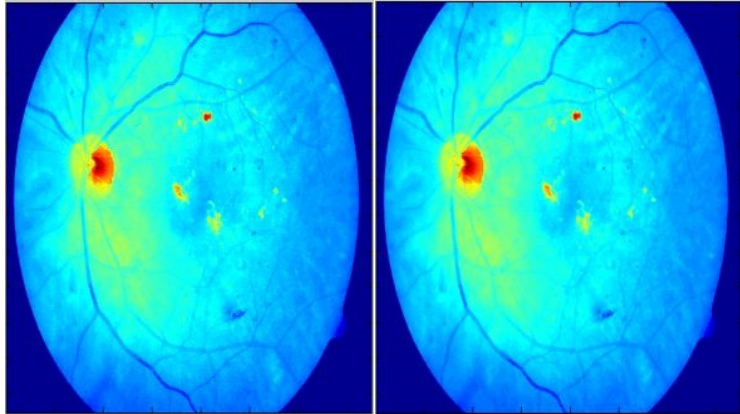

(a)

(b)

Fig4: (a) Component I of the HSI, result obtained by Median Filter

2.2.3 Adaptive Histogram Equalization (CLAHE) Adaptive histogram equalization (AHE) is a computer image processing technique used to improve contrast in images. It differs from ordinary histogram equalization in the respect that the adaptive method computes several histograms, each corresponding to a distinct section of the image, and uses them to redistribute the lightness values of the image. It is therefore suitable for improving the local contrast of an image and bringing out more detail (figure 4).

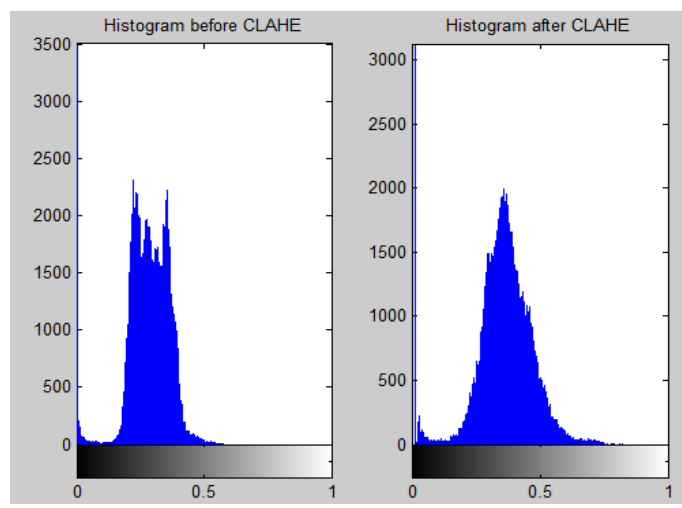

Fig 4: Adaptive Histogram Equalization

I component of the resulting median filtering and histogram equalization will be combined with the $\mathrm{H}$ and $\mathrm{S}$ components, all of which components will be processed again in the RGB color space (Figure 5).

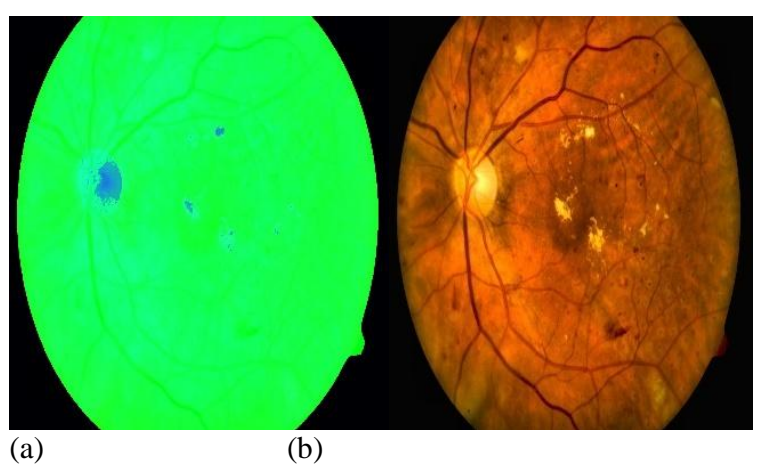

Fig 5: (a) Image after CLAHE, (b) Final image after preprocessing 


\subsection{Detection of the optic disc}

Barrett et al. [10] propose applying a Hough transform in order to locate the optic disc.

The Hough transform technique is able to find geometric shapes in an image. Objects of geometric shapes may be detected by converting the equation of the object into a Hough space parameter equation. For example, a line and a circle can be represented in Hough Space by:

$$
\begin{aligned}
& \text { Line: } \quad x_{i} \cos \theta+y_{i} \sin \theta=\rho \\
& \text { Circle: }(x i-a)^{2}+(y i-b)^{2}=r^{2}
\end{aligned}
$$

The line has two parameters in Hough space, the angle $\theta$ and length $\rho$ of the line. On the other hand the circle has three parameters in Hough space, the center $(a, b)$ and the radius $r$ of the circle. The optic disc has an approximately circular shape, therefore the Hough transform can be used to detect the optic disc. With the optic disc radius fixed in Hough parameter space, the search for a circular object becomes a two-dimensional problem.

This method finds the circular shape with fixed radius in a thresholded edge image of the fundus. To detect edges of all possible orientations at each pixel in an image compass edgedetection with aSobel kernel is applied. The maximal response of the Sobel kernel for each orientation is retained. On this edge map of the retinal surface a single threshold is applied to obtain a binary edge map (Figure 6,7).
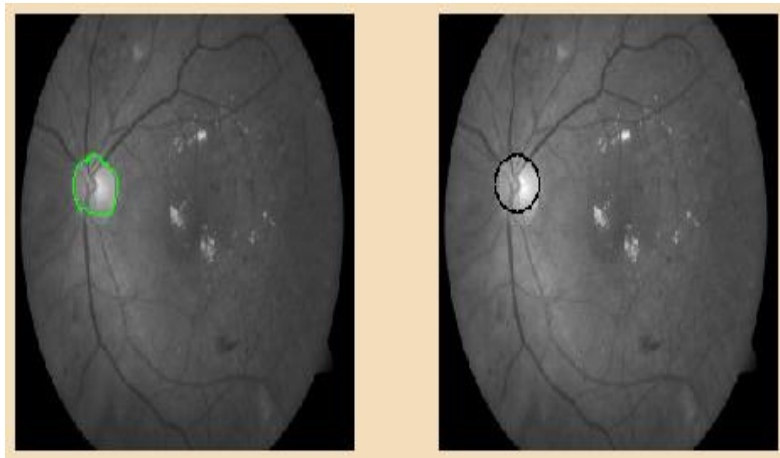

(a)

(b)

Fig 6: (a) Contour of Hough transform. (b) Circular approximation of the OD contour.

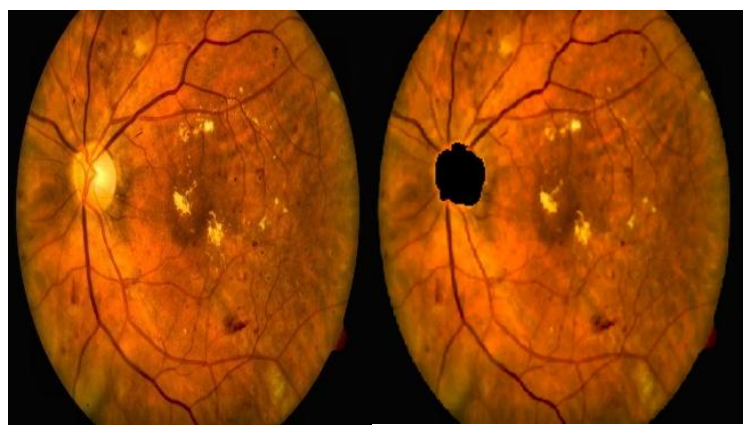

Fig 7: Optic disc extraction

\subsection{Segmentation using Graph Cuts}

Within this part contains exudates segmentation using graph theory. Our goal is to the detection and extraction of exudates supervised manner by a region growing segmentation.

Boykov [11] provides a method for adapting the graph cut to image processing and in particular to the object segmentation. It builds a graph $G=\{V, E\}$ formed by nodes $V$ and edges $E$. Along with consideration of the pixels of the image as nodes ,two additional nodes which are the s(source) for object and the $\mathrm{t}$ (terminal) for the background are considered (figure 8).

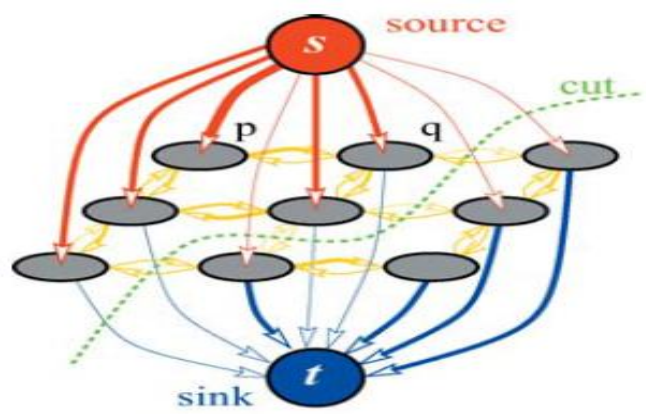

Fig 8: Graph cut segmentation of a $3 \times 3$ image

Graph Cuts finds the optimal solution to a binary problem. However when each pixel can be assigned many labels, finding the solution can be computationally expensive. For the following type of energy, a serie of graph cuts can be used to find a convenient local minimum:

$$
E(f)=\sum_{p \in P} D p(i p, f p)+\sum_{p \in P} V p, q(f p, f q)
$$

The first term in equation (7) is known as the data term. It ensures that the current labeling $\mathrm{f}$ is coherent with the observed data ip. It penalizes a label fp to pixel $\mathrm{p}$ if it is too different with the observed data ip. The second term is known as the smooth term. It ensures that the overal labeling $\mathrm{f}$ is smooth. It penalizes two neighboring labels fp and fq if they are too different.

In our work it is desired to cut a minimum for a graph with more than two terminal nodes. For this we chose the alphaexpansion algorithm.

\subsubsection{Alpha-Expansion}

The main idea of the alpha-expansion algorithm [12] is to successively segment all alpha and non-alpha pixels with graph cuts and the algorithm will change the value of alpha at each iteration. The algorithm will iterate through each possible label for alpha until it converges (figure 9).

At each iteration, the alpha region $\mathrm{P}_{\alpha}$ can only expand. This changes somehow the way to set the graph weights. Also when two neighboring nodes does not currently have the same label, an intermediate node is inserted and links are weighted so they are relative to the distance to the alpha label(figure $10)$. 


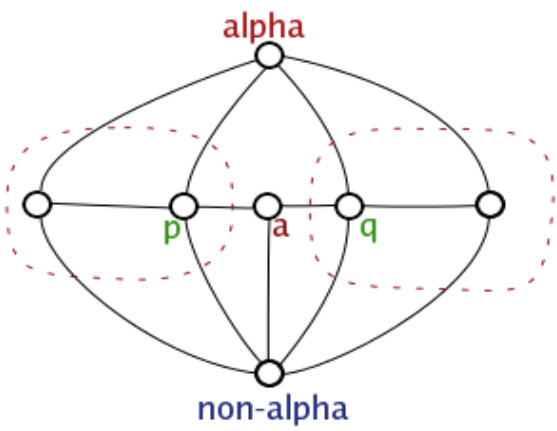

Fig 9: Alpha-expansion graph setting.

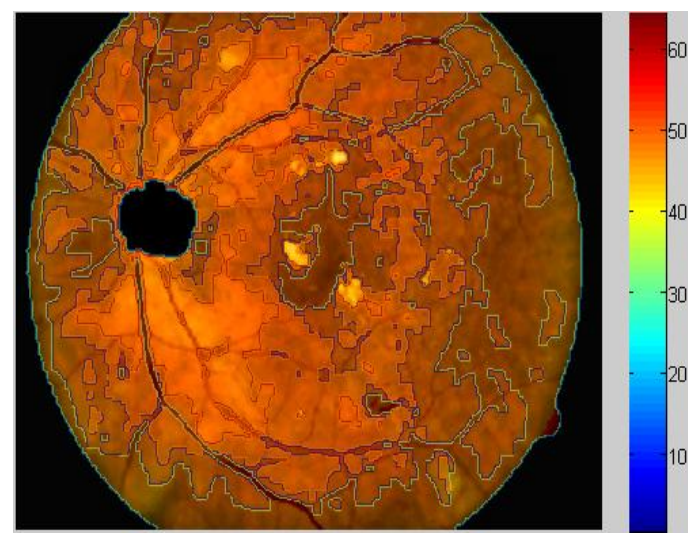

Fig 10: segmentation of alpha-expansion.

\subsubsection{Creating classes from segments}

In this step we will create classes from segments we obtained the previous stage. The result is shown in the following (figure 11):
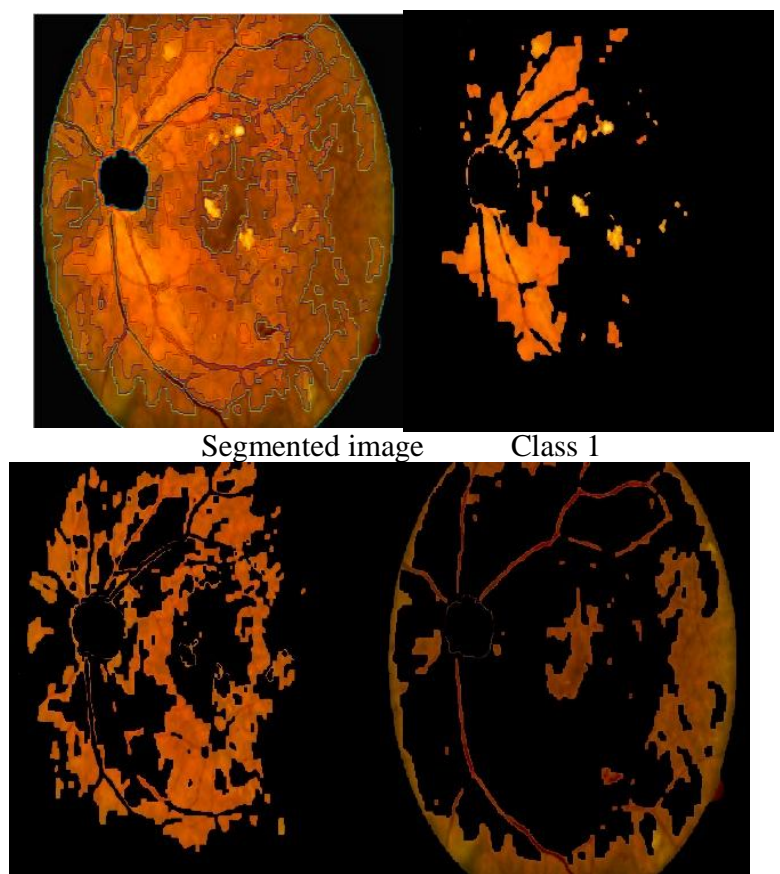

Class 2

Class 3

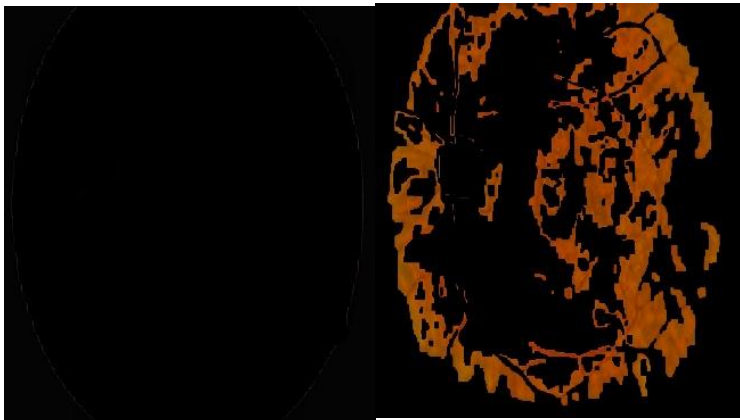

Class 4

Class 5

Fig 11: classes from segments.

\subsubsection{Selection and binarization of candidate} image.

In this section, we will select and binarized the image candidate (figure 12 (b)) knowing that the exudates are homogeneous in them color property and are characterized by a strong and brightness contrast comparable to other image. The result is indicated in the figure 12 (b).

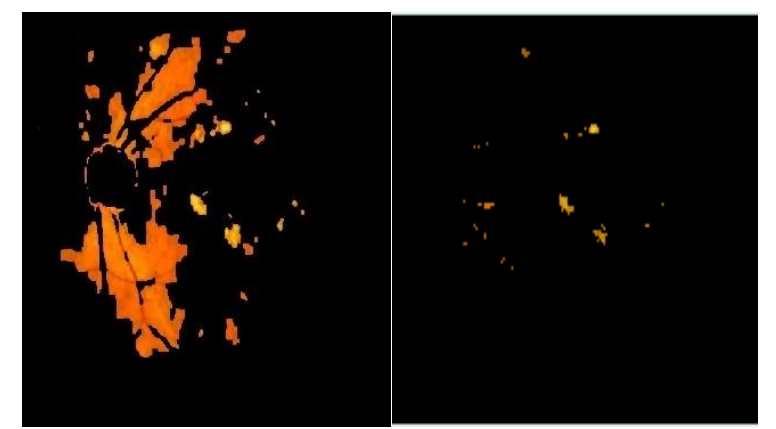

(a)

(b)

Fig 12: (a) Image candidate, (b) Final segmentation result of exudat

\subsubsection{Classification using neural network}

The representation of images is an important step in the recognition phase. It must be invariant to geometric transformations (rotation, translation and scale factor) and robust to various disturbances (noise, dimming, etc).

The representation we have adopted in this project is based on Hu moment applied to binary images.

Based on normalized central moments, $\mathrm{Hu}$ introduced seven moment invariants:

$$
\begin{aligned}
& \emptyset_{1}=\mu_{20}+\mu_{02} \\
& \begin{aligned}
& \emptyset_{2}=\left(\mu_{20}-\mu_{02}\right)^{2}+4 \mu_{11}^{2} \\
& \emptyset_{3}=\left(\mu_{30}-3 \mu_{12}\right)^{2}+\left(3 \mu_{21}-\mu_{03}\right)^{2} \\
& \emptyset_{4}=\left(\mu_{30}+\mu_{12}\right)^{2}+\left(\mu_{21}+\mu_{03}\right)^{2} \\
& \emptyset_{5}=\left(\mu_{30}-3 \mu_{12}\right)\left(\mu_{30}+\mu_{12}\right)\left[\left(\mu_{30}+\mu_{12}\right)^{2}\right. \\
&\left.-3\left(\mu_{21}+\mu_{03}\right)^{2}\right] \\
&+\left(3 \mu_{21}-\mu_{03}\right)\left(\mu_{21}\right. \\
&\left.+\mu_{03}\right)\left[3\left(\mu_{30}+\mu_{12}\right)^{2}-\left(\mu_{21}+\mu_{03}\right)^{2}\right] \\
& {\left[\left(\mu_{30}+\mu_{12}\right)^{2}-\left(\mu_{21}+\mu_{03}\right)^{2}\right] } \\
&+4 \mu_{11}\left(\mu_{30}+\mu_{12}\right)\left(\mu_{21}+\mu_{03}\right)
\end{aligned} \\
& \emptyset_{6}=\left(\mu_{20}-\mu_{02}\right)
\end{aligned}
$$




$$
\begin{aligned}
\emptyset_{7}=\left(3 \mu_{21}-\mu_{03}\right) & \left(\mu_{30}+\mu_{12}\right)\left[\left(\mu_{30}+\mu_{12}\right)^{2}\right. \\
& \left.-3\left(\mu_{21}+\mu_{03}\right)^{2}\right] \\
& -\left(\mu_{30}-3 \mu_{12}\right)\left(\mu_{21}\right. \\
& \left.+\mu_{03}\right)\left[3\left(\mu_{30}+\mu_{12}\right)^{2}-\left(\mu_{21}+\mu_{03}\right)^{2}\right]
\end{aligned}
$$

The seven moment invariants are useful properties of being unchanged under image scaling, translation and rotation.

The feature vectors obtained above are classified into normal or abnormal using Neural Networks Classifier (Table 1). It is one of the simplest but widely used machine learning algorithm. An Object is classified based on the distance from its neighbor.

Table 1. Construction of neural network

\begin{tabular}{|c|c|c|c|}
\hline Descriptor & $\begin{array}{c}\text { Number of } \\
\text { neurons in } \\
\text { input layer }\end{array}$ & $\begin{array}{c}\text { Number of } \\
\text { neurons in } \\
\text { hidden layer }\end{array}$ & $\begin{array}{c}\text { Number of } \\
\text { neurons in } \\
\text { output layer }\end{array}$ \\
\hline $\mathrm{Hu}$ & 8 & 8 & 1 \\
\hline
\end{tabular}

\section{RESULTS AND DISCUSSIONS}

\subsection{RGB to HSI conversion}

First, we convert RGB color space image to HSI space beginning with normalizing RGB.

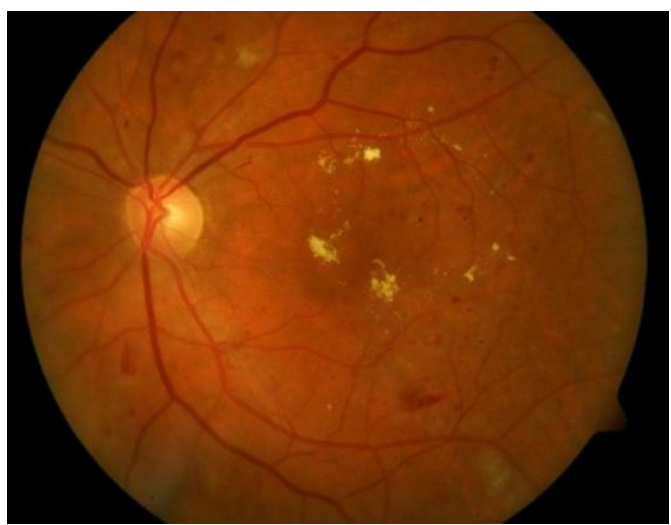

Fig 13 : Original Image (RGB)

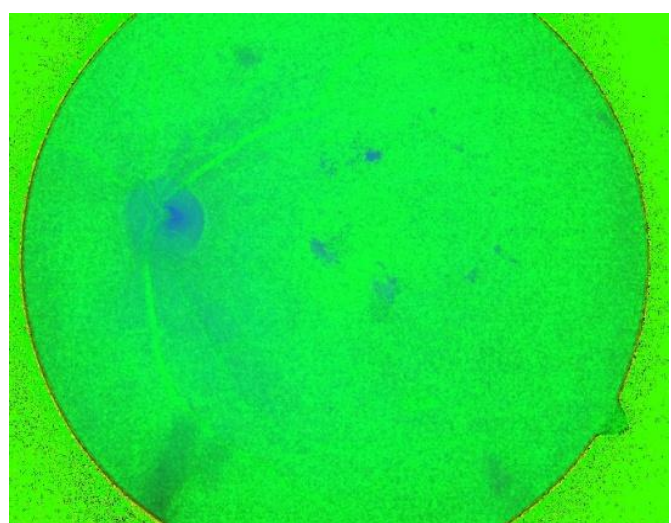

Fig 14 : HSI space

\subsubsection{Median Filtering:}

Intensity image is median filtered to remove noise.

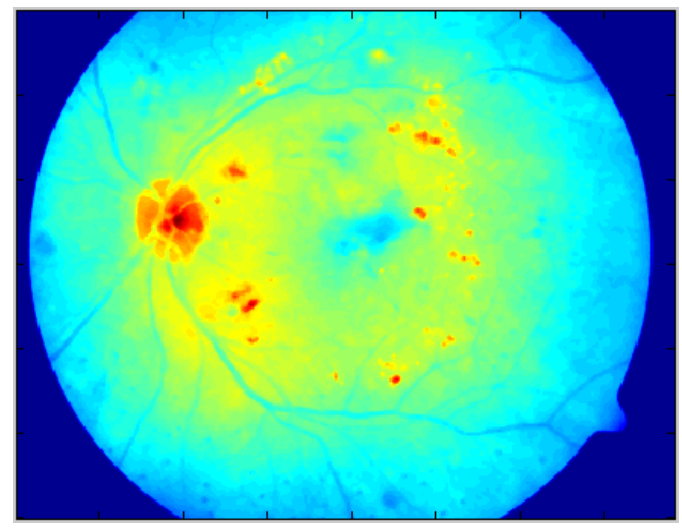

Fig 15: result obtained by Median Filter

\subsubsection{Adaptive Histogram Equalization}

CLAHE was originally developed for medical imaging and has proven to be successful for enhancement of low-contrast images.

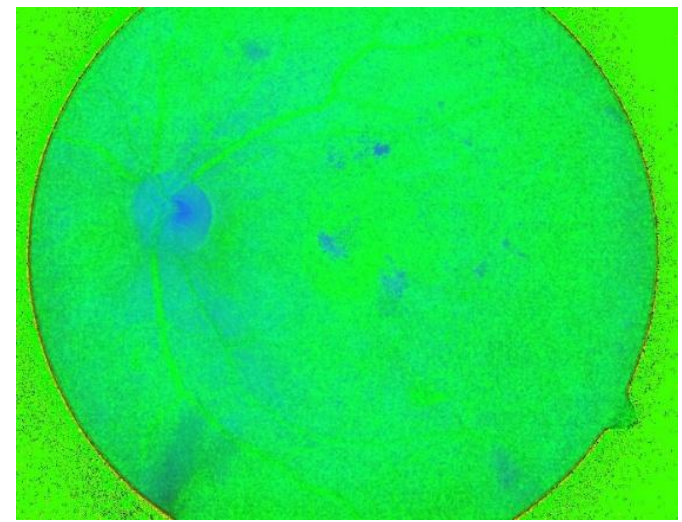

Fig 16: CLAHE

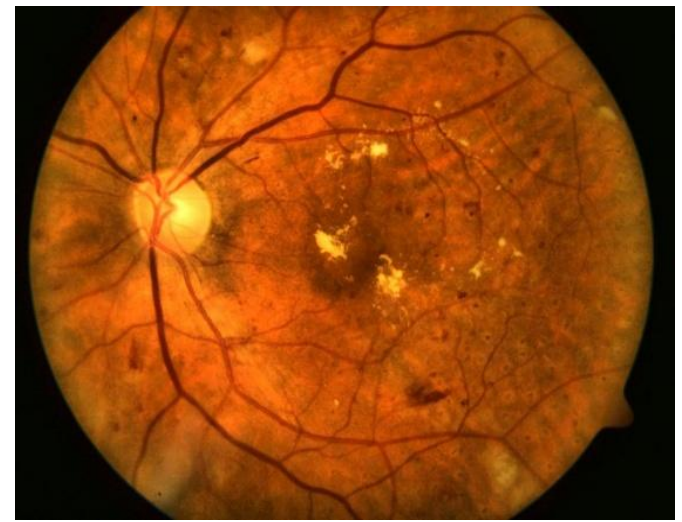

Fig 17: final image after pre-processing

\subsubsection{Optic disc extraction}

Hough transform is applied to histogram equalized image. Optic disc is detected. 


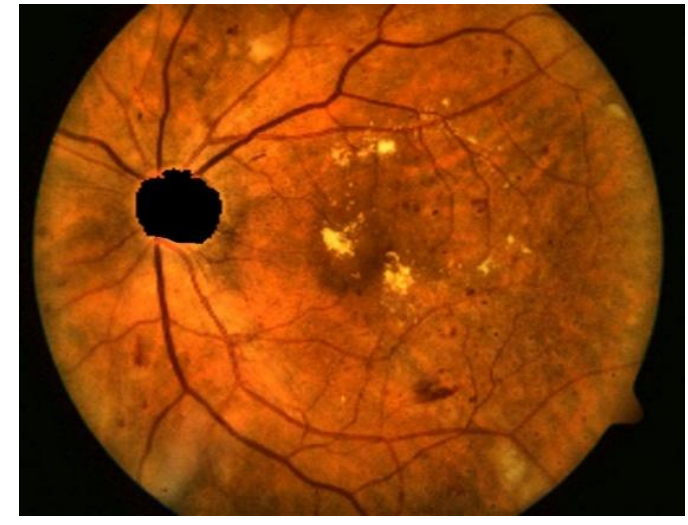

Fig 18: Optic Disk Eliminated

\subsubsection{Exudate Detection}

The detection and extraction of exudates using graph cut.

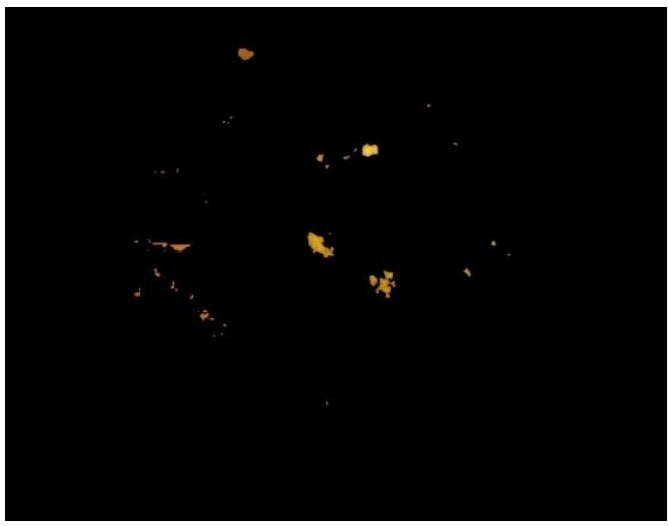

Fig 19: Exudates

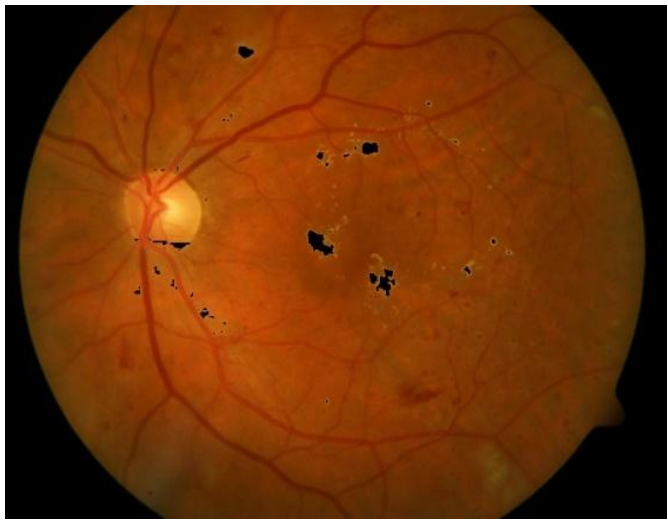

Fig 20: Our exudate detection result.

The images are classified as normal or abnormal using the neural network classifier.

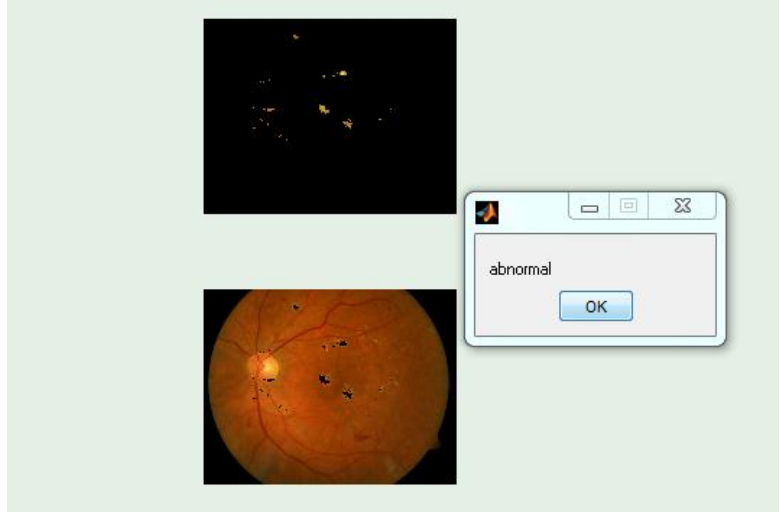

Fig 21: neural network classifier result.

In medical diagnosis, the medical input data is usually classified into two classes, where the disease is either present or absent. The classification accuracy of the diagnosis is assessed using the Sensitivity, Specificity, Precision, Accuracy and F-measure. Following the practices in the medical research, the fundus images related tothe diabetic retinopathy are evaluated by using sensitivity and specificity perimage basis. Sensitivity is the percentage of abnormal fund uses classified as abnormal, and specificity is the percentage of normal fundus classified as normal by the screening. These parameters are computed as:

$$
\begin{aligned}
& \text { Sensitivity }=\frac{\mathrm{TP}}{\mathrm{TP}+\mathrm{FN}}(9) \\
& \text { Specificity }=\frac{\mathrm{TN}}{\mathrm{TN}+\mathrm{FP}} \quad(10) \\
& \text { Precision }=\frac{\mathrm{TP}}{\mathrm{TP}+\mathrm{FP}} \\
& \text { Accuracy }=\frac{\mathrm{TP}+\mathrm{TN}}{\mathrm{TP}+\mathrm{TN}+\mathrm{FP}+\mathrm{FN}} \\
& \mathrm{F}-\text { measure }=\frac{2 * \mathrm{TP}}{2 * \mathrm{TP}+\mathrm{FN}+\mathrm{FP}}
\end{aligned}
$$

Table 2. Contingency table or confusion

\begin{tabular}{|c|c|c|}
\cline { 2 - 3 } \multicolumn{1}{c|}{} & Condition positive & Condition Negative \\
\hline $\begin{array}{c}\text { Test } \\
\text { Outcome } \\
\text { Positive }\end{array}$ & $\begin{array}{c}\text { True Positive } \\
\text { (TP) }\end{array}$ & $\begin{array}{c}\text { False Positive } \\
\text { (FP) }\end{array}$ \\
\hline $\begin{array}{c}\text { Test } \\
\text { Outcome } \\
\text { Negative }\end{array}$ & $\begin{array}{c}\text { False Negative } \\
\text { (FN) }\end{array}$ & $\begin{array}{c}\text { True Negative } \\
\text { (TN) }\end{array}$ \\
\hline
\end{tabular}

Where TP is the number of abnormal fundus images found as abnormal, $\mathrm{TN}$ is the number of normal fundus images found asnormal, FP is the number of normal fundus images found as abnormal (false positives) and FN is the number of abnormal fundus images found as normal (false negatives) (Table 2)..

To evaluate the performance of our approach, we compared our results with the performance of ophthalmologist. This 
approach is to measure the correctness of the algorithms at the pixel level. 46 retinal images were processed by steps proposed in the methodology section. Table 3 shows Sensitivity, Specificity, Precision, Accuracy and F-measure of our technique.

Table 3. Performance for Exudates

\begin{tabular}{|l|l|l|l|l|}
\hline $\begin{array}{l}\text { Sensitivi- } \\
\text { ty }\end{array}$ & $\begin{array}{l}\text { Specifici- } \\
\text { ty }\end{array}$ & Precision & $\begin{array}{l}\text { F- } \\
\text { measure }\end{array}$ & Accuracy \\
\hline $95 \%$ & $96,65 \%$ & $95 \%$ & $95 \%$ & $95,15 \%$ \\
\hline
\end{tabular}

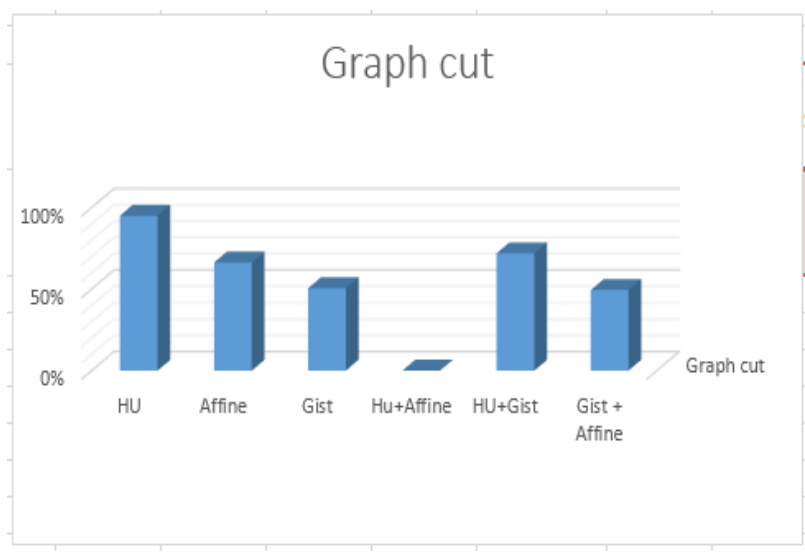

Fig 22: Recognition result by graph cut

By analyzing the graph (Figure 22) we find that the best combination of descriptors is that of graph cut with a descriptor vector composed of moment of $\mathrm{Hu}$ and GIST.

\section{CONCLUSIONS}

In this paper, a fast method of segmentation and recognition of exudates for diabetic retinopathy based on graph cut and Neural Network is proposed. This approach will be used to improve the precision of the diagnosis of the diabetes retinopathy before the stage of complications. We started in a first step with a preprocessing operation to improve image quality by eliminating defects caused by lighting and acquisition processes. In the second step the optic disc disrupts the automatic detection. For this we segmented to be removed. In the third step, the segmentation of graph cuts are used in order to detect exudates regions. Finally, the neural network gives better results with a feature extraction of images by descriptors and $\mathrm{Hu}$ moment of GIST. The final results are compared quantitatively with a manual exudates segmentation produced by an expert in ophthalmology. Performances of our method are measured by Specificity $95 \%$, sensitivity $96,65 \%$ value and accuracy $95,15 \%$.

\section{REFERENCES}

[1] K. V. Kauppi T, Kämäräinen J-K, Lensu L, Sorri I, Raninen A, "DIARETDB1: diabetic retinopathy database and evaluation protocol. In: Medical image understanding and analysis (MIUA).",ed, 2007.

[2] Gardner GG, Keating D, Williamson TH, Elliott AT, "Automatic detection of diabetic retinopathy using an artificial neural network: a screening tool", British journal of Ophthalmology, pp. 940-944, 1996.

[3] Sinthanayothin C, Boyce JF, Williamson TH, Cook HL, Mensah E, Lal S, "Automated detection of diabetic retinopathy on digital fundus image", Journal of Diabetic Medicine, vol 19, pp 105-112,2002.

[4] C.I. Sanchez, R. Hornero, M.I. Lopez, and J.Poza, Retinal image analysis to detect and quantify lesions associated with diabetic retinopathy,IEEE Conf. on Engineering in Medicine and Biology Society, vol.1, pp.1624-1627, 2004

[5] Kavitha, D. and S.D. Shenbaga. Automatic detection of optic disc and exudates in retinal images. Proceedings of the International Conference on Intelligent Sensing and Information Processing, Jan. 4-7, IEEE Xplore Press, pp: 502-506.2005.

[6] AkaraSopharak, BunyaritUyyanonvara, Sarah Barman, "Automatic Exudate Detection from Non-dilated Diabetic Retinopathy retinal images using Fuzzy CMeans Clusterg" Journal of Sensors, vol.9, No. 3, pp 2148- 2161, March 2009.

[7] D. Welfer, J. Scharcanski, D.R. Marinho, "A coarse-tofine strategy for automatically detecting exudates in color eye fundus images," Computerized Medical Imaging and Graphics, vol. 34, pp. 228-235, 2010.

[8] AkaraSopharak, Mathew N. Dailey, BunyaritUyyanonvara, Sarah Barman, Tom Williamson, Yin Aye Moe, "Machine Learning approach to automatic Exudates detection in retinal images from diabetic patients", Journal of Modern optics, Vol. 57, No. 2, pp. 124-135,Nov 2011.

[9] DeepashreeDevaraj, Dr.Prasanna KumarS.C.,Manisha, "Automatic Exudate detection for the diagnosis of Diabetic Retinopathy",International Journal of Innovative Research and Studies,Volume 2 Issue 5- May 2013,Page No 657-669.

[10] S.F. Barrett, E. Naess, T. Molvik., "Employing the hough transform to locate the optic disk", Biomedical Sciences Instrumentation 37 (2001) 81-86.

[11] Boykov, Y., Veksler, O., and Zabih, R. Fast approximate energy minimi-zation via graph cuts. IEEE Trans. Pattern Anal. Mach. Intell. 23, 11 (2001), 1222\{1239.

[12] Boykov, Y., and Jolly, M.-P. Interactive graph cuts for optimal boundary and region segmentation of objects in n-d images. In International Conference on Computer Vision (ICCV) (2001), pp. 105\{112\} 